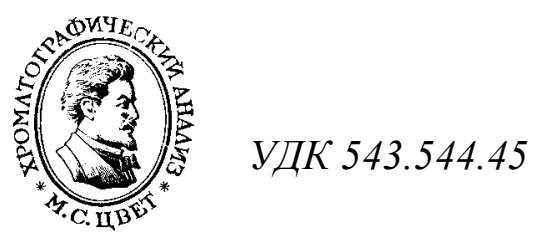

\title{
Особенности использования дополнительных стандартов в количественном хроматографическом анализе
}

\author{
Зенкевич И.Г., Прокофьев Д.В. \\ Санкт-Петербургский государственньй университет, Институт химии, Санкт-Петербург \\ Поступила в редакцию 31.01.2017 г.
}

\begin{abstract}
Основной причиной низкой воспроизводимости (повторяемости) результатов количественного хроматографического анализа являются трудно учитываемые частичные потери проб в процессе их дозирования, особенно значительные в газовой хроматографии. Компенсация этого фактора возможна в результате замены абсолютных площадей хроматографических пиков их отношениями к площадям пиков дополнительных стандартов. Такой прием наиболее эффективен в способах внешнего стандарта и стандартной добавки, но несколько более трудоемок в методе абсолютной градуировки.

Обсуждаются требования к дополнительным стандартам, а именно к выбираемым для этого соединениям, к стадиям и операциям анализа, а также к алгоритмам обработки экспериментальных данных. Специально отмечено, что для дополнительных стандартов они не идентичны требованиям к внутренним стандартам в одноименном способе. В качестве дополнительных стандартов можно использовать входящие в состав образцов растворители.
\end{abstract}

Ключевые слова: Количественный хроматографический анализ, случайная составляющая погрешностей определений, дополнительные стандарты, особенности использования.

\section{Additional standards in quantitative chromatographic analysis features of application}

\author{
Zenkevich I.G., Prokof'ev D.V. \\ St. Petersburg State University, Institute for Chemistry, St. Petersburg
}

The principal reason for low reproducibility of chromatographic peak areas and, hence, the final results of quantitation, seems to be the partial losses of samples during their injection. The method of its compensation is considered; it implies replacing the absolute peak areas $\left(S_{\mathrm{i}}\right)$ with their ratios to peak areas of additional standards $\left(S_{\mathrm{i}} / S_{\text {add.stand. }}\right)$, which should be preliminary added to the samples of interest.

The features of the application of additional standards are considered. They are not identical to those of internal standards in the same-named method of quantification. The principal differences are i) no restrictions on the chemical origin of additional standards, ii) no information on their concentrations and calibration coefficients, like it should be known for internal standards. Sometimes the equality of their amounts in two samples (external standard), or proportionality in few samples (absolute calibration) should be provided. The solvents of samples of interest can be used as additional standards. This approach allows dilution of samples (without control of the dilution ratio), as well as the variations of injected amounts.

The primary processing of experimental data includes measuring peak areas, calculation of ratios $\left(S_{\mathrm{i}} / S_{\text {add.stand. }}\right)$, their averaging, and only after that using the target relations. The modification considered is recommended preferably for methods of external standard, standard addition, and, little less convenient, absolute calibration.

Keywords: Quantitative chromatographic analysis, repeatability of results, additional standards, features of application. 


\section{Введение}

К основным способам количественного хроматографического анализа относят методы внешнего стандарта (I), абсолютной градуировки (II), внутреннего стандарта (III), стандартной добавки (IV) и внутренней нормализации (V). «Классические» варианты этих методов [2-5], прежде всего (I-II и IV), основаны на измерении и использовании в расчетах абсолютных площадей хроматографических пиков целевых компонентов $\left(S_{\mathrm{i}}\right)$ и, при необходимости, стандартов $\left(S_{\text {станд }}\right)$. Именно воспроизводимость (повторяемость) площадей пиков определяет случайную составляющую погрешностей результатов. Из различных факторов, снижающих их воспроизводимость, основным, особенно в газовой хроматографии, представляются неконтролируемые частичные потери проб в процессе дозирования. В высокоэффективной жидкостной хроматографии и капиллярном электрофорезе этот фактор менее значим, так как дозирование не связано с вводом проб в нагретые участки хроматографических систем в зонах повышенного давления. Не столько для минимизации таких потерь, сколько для повышения их воспроизводимости за счет уменьшения влияния «человеческого фактора» в последнее время часто рекомендуют использовать автоматические дозирующие устройства $[5,6]$. Такие устройства, безусловно, гарантируют воспроизводимое время нахождения шприцев в нагретых зонах испарителей, но не ликвидируют возможные потери проб в самих шприцах.

Альтернативный способ повышения воспроизводимости результатов хроматографического анализа предполагает не устранение, а компенсацию возможных потерь проб на стадии их дозирования за счет замены абсолютных площадей пиков их отношениями к площадям пиков некоторых стандартов, поскольку такие отношения почти не зависят от искажений дозируемых количеств. Принципиально сразу же отметить, что выбираемые для этого стандарты не идентичны стандартам, используемым в одноименном методе внутреннего стандарта (III), так что для исключения возможных неоднозначностей терминологии авторы настоящей работы остановились на термине дополнительные стандарты. В наибольшей степени замена $\left(\mathrm{S}_{\mathrm{i}}\right)$ отношениями $\left(\mathrm{S}_{\mathrm{i}} / \mathrm{S}_{\text {доп.станд }}\right)$ целесообразна в методах внешнего стандарта $(\mathrm{I})$, стандартной добавки (IV) и абсолютной градуировки (II). В расчетных соотношениях метода внутреннего стандарта (III) уже присутствуют отношения $\left(\mathrm{S}_{\mathrm{i}} / \mathrm{S}_{\text {станд }}\right)$, а в методе внутренней нормализации $(\mathrm{V})$ при вычислении отношений $\left(\mathrm{S}_{\mathrm{i}} / \Sigma \mathrm{S}_{\mathrm{i}}\right)$ роль стандарта формально играет совокупность всех регистрируемых на хроматограмме компонентов. Следовательно, применение дополнительных стандартов в двух последних случаях не актуально.

Парадоксальность таких рекомендаций (использования относительных площадей пиков вместо абсолютных величин) состоит в том, что отдельные элементы этого подхода, фактически, известны. Под названием «Объединенный метод добавки и внутреннего стандарта» они рассмотрены, например, в методическом руководстве [7]. Однако при этом, как ни удивительно, в большинстве монографий и учебных пособий (включая цитированные выше руководства [2-5]) они даже не упоминаются. Единичные примеры использования такого подхода отмечены лишь в последнее время (см. [8-11]), но его систематическая характеристика была начата только в наших публикациях [12-15].

Для наглядной характеристики преимуществ такого подхода был выбран такой прием, как специальное моделирование низкой повторяемости результатов изза неконтролируемых частичных потерь проб при их дозировании [12-15]. С этой целью использовали сильно изношенный и, следовательно, недостаточно гер- 
метичный микрошприц объемом 1 мкл. По аналогии с работами [12-15] авторы считают необходимым специально отметить, что характеристики шприцев никак не связаны с их торговыми марками, а определяются только сроками и условиями их предшествующей эксплуатации.

Часть результатов такого моделирования на примере раствора 50 мкл 1,2,4триметилбензола в $2 \mathrm{~cm}^{3} 2$-пропанола (площади пиков растворенного компонента и растворителя) приведены в Табл. 1. Однако подобная табличная форма представления данных не позволяет выявить взаимные корреляции площадей пиков разных компонентов и, по этой причине, недостаточно информативна. Для лучшей визуализации результатов первоначально были рекомендованы гистограммы [12], однако наиболее полезными оказались огибающие ломаные линии семейств точек в координатах «площадь пика - порядковый номер пробы», $S_{\mathrm{i}}(N)$ для разных компонентов образцов [15]. На рис. 1 приведен подобный график для компонентов раствора 1,2,4-триметилбензола в 2-пропаноле, построенный по результатам 22 вводов проб.

Таблица 1. Пример, иллюстрирующий низкую воспроизводимость площадей пиков компонента раствора (1,2,4-триметилбензол) и растворителя (2-пропанол) в результате их неконтролируемых потерь проб при дозировании

\begin{tabular}{|c|c|c|c|c|}
\hline Компонент & $\begin{array}{c}\text { Значения } S_{\mathrm{i}}(1 \leq i \leq 22), \\
\text { мВ } \times \text { мс }\end{array}$ & $\begin{array}{l}\text { Среднее } \\
\text { значение }\end{array}$ & $\begin{array}{c}\text { Стандартное } \\
\text { отклонение }\end{array}$ & $\begin{array}{c}\text { Коэффициент } \\
\text { вариации, } \\
\delta, \%\end{array}$ \\
\hline $\begin{array}{c}\text { 1,2,4-Триметил- } \\
\text { бензол }\end{array}$ & $\begin{array}{l}22078,34317,18522,15325, \\
36375,12214,39399,35770, \\
20207,15577,20408,23898, \\
29498,35870,3033,11932, \\
\begin{array}{l}36578,31578,4282, \\
6299,\end{array}\end{array}$ & 22107 & 11690 & $\delta=53$ \\
\hline $\begin{array}{r}\text { Растворитель } \\
\text { (2-пропанол) }\end{array}$ & $\begin{array}{lrr}359856, & 526551, & 287266, \\
241414, & 534680, & 197981, \\
580484, & 534427, & 311147, \\
242048, & 313836, & 363519, \\
440818, & 521985, & 53889, \\
190512, & 536548, & 470598, \\
75429, & 396328, & 105717, \\
117195 & & \end{array}$ & 336465 & 168136 & $\delta=50$ \\
\hline
\end{tabular}

В качестве дополнительных стандартов можно использовать любые как искусственно вводимые в анализируемые образцы вещества, так и уже присутствующие в них компоненты, в том числе растворители, несмотря на то, что их количества могут существенно превышать содержание целевых аналитов. Возможность использования растворителей в качестве внутренних стандартов фактически была предложена в работах С.В. Черепицы, в том числе [16-19]. В рассматриваемом примере в качестве дополнительного стандарта выбрали именно растворитель (2-пропанол). Легко визуально воспринимаемая идентичность контуров двух ломаных линий наглядно подтверждает единый для обоих компонентов проб источник случайных погрешностей, а именно стадию дозирования проб. Из этого следует, во-первых, возможность описания двух наборов площадей пиков уравнением линейной регрессии $S(1,2,4-$ триметилбензол $)=a S(2$-пропанол $)+b$. Параметры уравнения приведены в подписи к рис. 2; коэффициент корреляции составляет $r=0.9990$. Во-вторых, воспроизводимость отношений площадей пиков 


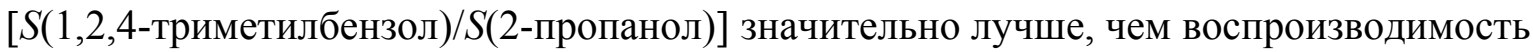
каждой из площадей по отдельности. Если относительные стандартные отклонения (коэффициенты вариации) площадей пиков каждого из компонентов достигают 50$53 \%$ (см. табл. 1), то аналогичная характеристика их отношений (табл. 2) составляет всего 3.5\%, т.е. в 14-15 раз меньше. На большем числе примеров [12-15] было показано, что повторяемость отношений площадей пиков аналитов и дополнительных стандартов в 6-38 раз лучше, чем исходных площадей, что обеспечивает высокую точность количественного хроматографического анализа даже при неприемлемо низкой воспроизводимости дозирования проб.

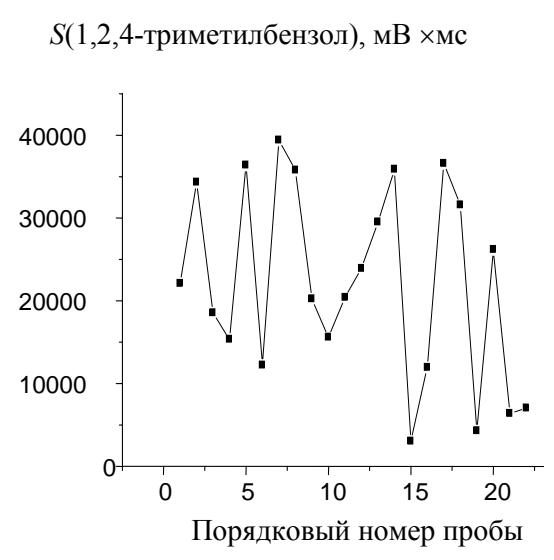

a

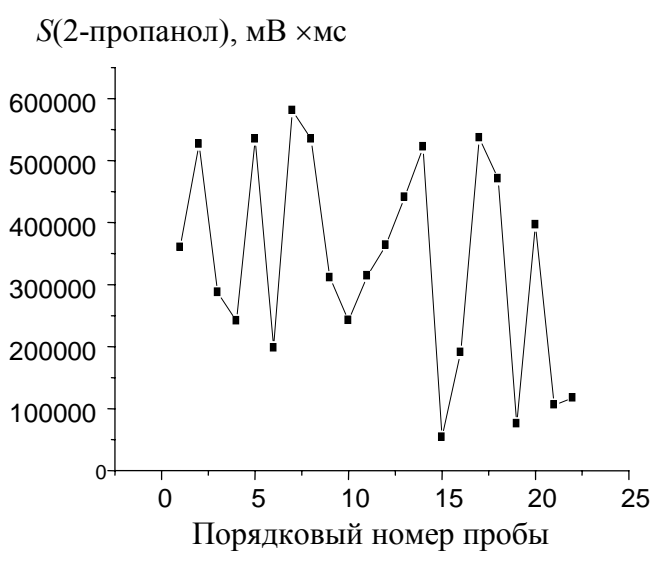

б

Рис. 1. Графическая иллюстрация разброса площадей хроматографических пиков компонента образца в результате неконтролируемых частичных потерь проб при их дозировании а) 1,2,4-триметилбензол, б) растворитель (2-пропанол). Идентичность контуров ломаных линий подтверждает единый для всех составляющих проб источник случайных погрешностей (соответствует данным таблицы 1).

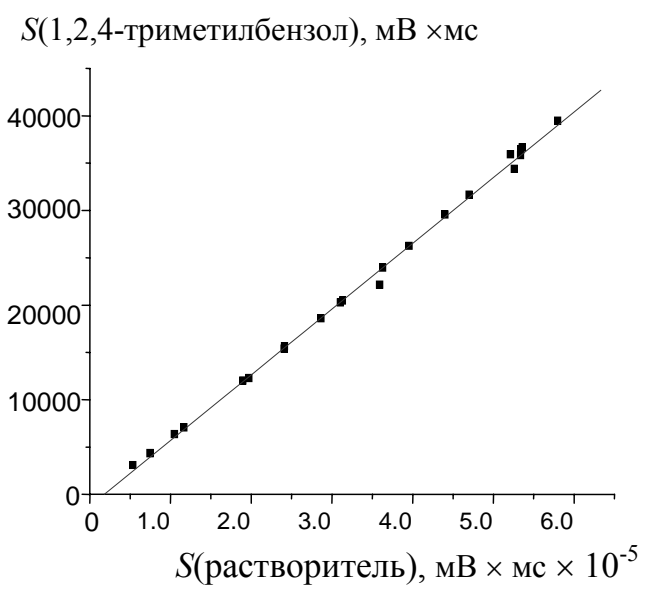

Рис. 2. Корреляция площадей пиков компонента образца и растворителя в условиях низкой воспроизводимости дозирования проб в хроматографическую колонку: $S(1,2,4-$-риметилбензол $)=a S(2$-пропанол $)+b$; Параметры уравнения линейной регрессии: $a=(69.45 \pm 0.07) \times 10^{-2}, b=-1261 \pm 264, r=0.9990, S_{0}=54$.

Важнейшим моментом рассматриваемого «дизайна» методов количественного хроматографического анализа, основанного на замене абсолютных площадей $\left(S_{\mathrm{i}}\right)$ отношениями $\left(S_{\mathrm{i}} / S_{\text {доп.станд }}\right)$, представляется выбор, свойства и способы использования дополнительных стандартов. Настоящая работа посвящена характеристике дополни- 
тельных стандартов и особенностей их применения в различных методах количественного газохроматографического анализа.

Таблица 2. Согласованные с табл. 1 данные, иллюстрирующие воспроизводимость

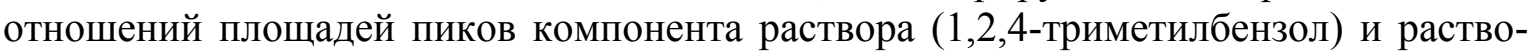
рителя (2-пропанол) вне зависимости от их разброса в результате их неконтролируемых потерь проб при дозировании

\begin{tabular}{|c|c|c|c|}
\hline $\begin{array}{c}\text { Отношения площадей пиков } \\
S_{\mathrm{i}} / S(\text { раств })_{\mathrm{i}}\end{array}$ & $\begin{array}{c}\text { Среднее } \\
\text { значение }\end{array}$ & $\begin{array}{c}\text { Стандартное } \\
\text { отклонение }\end{array}$ & $\begin{array}{c}\text { Коэффициент ва- } \\
\text { риации, } \delta, \%\end{array}$ \\
\hline $\begin{array}{c}0.061,0.065,0.064,0.063,0.068, \\
0.062,0.068,0.067,0.065,0.064,\end{array}$ & 0.066 & 0.02 & $\delta=3.5$ \\
$\begin{array}{c}0.065,0.066,0.067,0.069,0.063, \\
0.068,0.067,0.067\end{array}$ & & & \\
\hline
\end{tabular}

\section{Теоретическая часть}

Сравнительная характеристика внутренних и дополнительных стандартов. История подготовки предыдущих публикаций по рассматриваемому вопросу [12-15], показала, что одной из его главных проблем является несколько необычное предназначение дополнительных стандартов, особенно в сравнении с часто используемыми в хроматографической практике внутренними стандартами. Набор требований, предъявляемых к внутренним стандартам в одноименном способе количественного анализа, хорошо известен (см., например, руководства $[4,20])$ и включает, в том числе, образование гомогенных смесей с анализируемыми образцами, инертность в отношении их компонентов, сходство химической природы стандартов и аналитов, их параметров удерживания, а также небольшие различия их концентраций в образцах. Естественно, что количества стандартов, добавляемых к известным количествам образцов, должны быть известны, а градуировочные коэффициенты аналитов относительно таких стандартов - определены предварительно. Требования же к дополнительным стандартам лишь частично совпадают с перечисленными, а некоторые принципиально от них отличаются. Сравнительная характеристика внутренних и дополнительных стандартов по критериям, относящимся к выбираемым для этого веществам, к процессу анализа (стадиям и операциям), а также к процедурам обработки результатов приведена в табл. 3 .

Случаи несоответствия характеристик внутренних и дополнительных стандартов в табл. 3 выделены курсивом. Такие общие критерии как смешиваемость с образцами, стабильность в условиях хроматографического разделения и инертность в отношении других компонентов, безусловно, идентичны для стандартов обоих типов. Однако по такому критерию как химическая природа они уже могут отличаться. Оптимальным вариантом внутренних стандартов из-за сходства химической природы и гарантированного разделения с целевыми аналитами, как в условиях газовой (как с капиллярными, так и с насадочными колонками), так и обращенно-фазовой ВЭЖХ, представляются их гомологи. На химическую же природу дополнительных стандартов никаких ограничений нет; главным условием становятся приемлемые значения параметров удерживания (разделение с определяемыми компонентами образцов) и их регистрация выбранными детекторами. Выполнение такого условия не требует добавочных экспериментов, так как это можно сделать, пользуясь справочными величинами газохроматографических индексов удерживания [21]. Известные количества внутренних стандартов вводят в анализируемые образцы искусственно, поэтому они должны отсутствовать в их составе. В качестве же дополнительных 
стандартов можно выбирать любые компоненты, как присутствующие в образцах (в том числе растворители), так и добавляемые к ним искусственно.

Таблица 3. Сравнительная характеристика внутреннего и дополнительного стандартов

\begin{tabular}{|c|c|c|}
\hline Критерий сравнения & Внутренний стандарт & Дополнительный стандарт \\
\hline \multicolumn{3}{|c|}{ Характеристики самого стандарта } \\
\hline $\begin{array}{c}\text { Смешивается ли с анализи- } \\
\text { руемым образцом? }\end{array}$ & Да & Да \\
\hline $\begin{array}{c}\text { Стабильность в условиях } \\
\text { хроматографического разде- } \\
\text { ления и инертность в отно- } \\
\text { шении других компонентов }\end{array}$ & Да & Да \\
\hline Химическая природа & $\begin{array}{c}\text { Максимально соответствует } \\
\text { химической природе анали- } \\
\text { тов. Оптимальный вариант } \\
\text { стандартов - гомологи }\end{array}$ & $\begin{array}{c}\text { Любые детектируемые ком- } \\
\text { поненты, приемлемые по } \\
\text { параметрам удерживания }\end{array}$ \\
\hline $\begin{array}{c}\text { Присутствие в анализиру- } \\
\text { емом образце }\end{array}$ & Отсутствует & Может присутствовать \\
\hline $\begin{array}{c}\text { Положение пика на хромато- } \\
\text { грамме }\end{array}$ & $\begin{array}{c}\text { Максимально близко к пику } \\
\text { аналита при условии полного } \\
\text { разделения }\end{array}$ & $\begin{array}{c}\text { Теоретически нет ограниче- } \\
\text { ний }\end{array}$ \\
\hline $\begin{array}{l}\text { Возможно ли использование } \\
\text { растворителя как стандарта? }\end{array}$ & Да & Да \\
\hline \multicolumn{3}{|c|}{ Характеристики процедуры анализа } \\
\hline $\begin{array}{c}\text { Возможность разбавления } \\
\text { анализируемых образцов }\end{array}$ & Да & Да \\
\hline $\begin{array}{c}\text { Возможность варьирования } \\
\text { объема дозируемых проб }\end{array}$ & Да & Да \\
\hline $\begin{array}{c}\text { Необходимость точного за- } \\
\text { дания массы или концентра- } \\
\text { ции } \\
\text { Альтернативная формули- } \\
\text { ровка: содержание в образце }\end{array}$ & $\begin{array}{c}\text { Известно; сравнимо с содер- } \\
\text { жанием аналита }\end{array}$ & $\begin{array}{c}\text { Не нужно (в различных спо- } \\
\text { собах количественного ана- } \\
\text { лиза это условие имеет свои } \\
\text { особенности) } \\
\text { Может быть неизвестным }\end{array}$ \\
\hline $\begin{array}{c}\text { Необходимо ли предварите- } \\
\text { льное определение градуиро- } \\
\text { вочных коэффициентов? }\end{array}$ & Да & $\mathrm{Hem}$ \\
\hline \multicolumn{3}{|c|}{ Процедура обработки результатов } \\
\hline $\begin{array}{c}\text { Первичная обработка площа- } \\
\text { дей пиков результатов па- } \\
\text { раллельных определений }\end{array}$ & $\begin{array}{c}\text { Определение площадей пи- } \\
\text { ков } \mathrm{S}_{\mathrm{i}} \text { и } \mathrm{S}_{\text {внутр-станд }} \mathrm{c} \text { последу- } \\
\text { ющим вычислением отноше- } \\
\text { ний }\left(\mathrm{S}_{\mathrm{i}} / \mathrm{S}_{\text {внутр-станд }}\right) \text { и их усред- } \\
\text { нением } \\
\end{array}$ & $\begin{array}{c}\text { Определение площадей пи- } \\
\text { ков } \mathrm{S}_{\mathrm{i}} \text { и } \mathrm{S}_{\text {доп-станд }} \text { с последую- } \\
\text { щим вычислением отноше- } \\
\text { ний }\left(\mathrm{S}_{\mathrm{i}} / \mathrm{S}_{\text {доп-станд }}\right) \text { и их усред- } \\
\text { нением } \\
\end{array}$ \\
\hline
\end{tabular}

Среди критериев, относящихся к процедуре анализа, для внутренних и дополнительных стандартов есть как совпадения, так и различия (см. табл. 3). Образцы, содержащие как внутренние, так и дополнительные стандарты можно разбавлять инертными растворителями, не контролируя степень разбавления, а также варьировать объемы дозируемых проб. Для внутренних стандартов необходимо точное задание их концентраций, тогда как в случае дополнительных стандартов это не требуется. Однако особенности реализации этого условия в разных способах количествен- 
ного анализа отличаются (см. далее). Так, например, в способе внешнего стандарта необходимо обеспечить равенство концентраций дополнительных стандартов в двух образцах; при этом сами концентрации могут оставаться неизвестными. В способе стандартной добавки требуется пропорциональность количеств таких стандартов в нескольких пробах. Однако наиболее принципиальным отличием представляется следующее: использование внутренних стандартов для количественного анализа подразумевает необходимость определения градуировочных коэффициентов определяемых аналитов относительно стандартов. Предназначение же дополнительных стандартов состоит в замене абсолютных площадей пиков $\left(S_{\mathrm{i}}\right)$ отношениями $\left(S_{\mathrm{i}} / S_{\text {доп.станд }}\right)$, что не требует определения градуировочных коэффициентов.

Третья группа критериев сравнения стандартов разных типов - особенности процедуры обработки результатов, которые для внутренних и дополнительных стандартов принципиально не отличаются. В обоих случаях вычисляют отношения непосредственно измеряемых площадей пиков $\left(S_{\mathrm{i}} / S_{\text {внутр-станд }}\right)$ или $\left(S_{\mathrm{i}} / S_{\text {доп-станд }}\right)$ с их последующим усреднением.

Модификация расчетных соотношений основных способов количественного хроматографического анализа при использовании дополнительных стандартов

Способ внешнего стандарта. «Традиционный» вариант этого способа подразумевает анализ двух образцов (обычно растворы): целевого (с неизвестной концентрацией аналита $C_{\mathrm{x}}$ ) и внешнего стандарта с заданным содержанием определяемого компонента $\left(C_{\mathrm{cr}}\right)$ и последующими вычислениями по простейшей пропорции:

$$
C_{x}=\frac{C_{c m} \cdot S_{x}}{S_{c m}}
$$

где $S_{\mathrm{x}}$ и $S_{\mathrm{c \tau}}-$ средние значения площадей пиков определяемого компонента и стандарта.

Использование формулы (1) подразумевает постоянство и равенство объемов

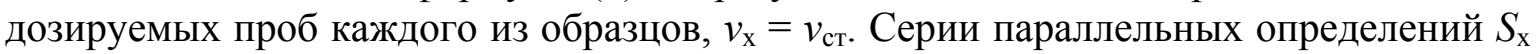
и $S_{\text {ст }}$ статистически независимы, поэтому средние значения этих площадей должны быть вычислены до подстановки в формулу (1). Поскольку усреднение экспериментальных данных важно для последующего изложения, то формулу (1) целесообразно уточнить, используя обычно принятые для обозначения средних величин $x=\left(\Sigma x_{\mathrm{i}}\right) / n$ символы $\langle x>$ :

$$
C_{x}=\frac{C_{c m} \cdot\left\langle S_{x}\right\rangle}{\left\langle S_{c m}\right\rangle}
$$

Наличие в формуле (1) отношения $\left(S_{\mathrm{x}} / S_{\mathrm{c \tau}}\right)$ не означает замену абсолютных площадей пиков относительными, так как они определены для разных образцов.

Для оценки случайной составляющей относительной погрешности результатов определений методом внешнего стандарта $\left(\delta C_{\mathrm{x}}\right)$ используют следующее соотношение:

$$
\delta C_{x} \approx\left[\delta S_{x}^{2}+\delta S_{c m}^{2}\right]^{1 / 2},
$$

где $\delta$ - символ относительных стандартных отклонений, $\delta(A)=s(A) /<A>$.

Именно эта случайная составляющая погрешностей результатов «традиционного» варианта способа внешнего стандарта чрезвычайно чувствительна к неконтролируемым потерям проб при их дозировании. Использование дополнительных стандартов предназначено как раз для компенсации подобных погрешностей. Практическая реализация модифицированного варианта метода внешнего стандарта предполагает весьма несложную вспомогательную операцию, а именно добавку равных количеств дополнительного стандарта к равным объемам растворов анализируемого 
образца $(x)$ и внешнего стандарта (ст) для соблюдения условия $M_{\text {доп.ст(х) }}=M_{\text {доп.ст(ст) }}$.

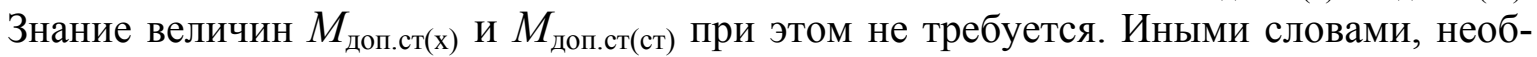
ходимо обеспечить равенство концентраций дополнительного стандарта в двух образцах; при этом сами концентрации могут оставаться неизвестными. Удобнее всего дозировать стандарты в виде равных объемов жидкостей или их растворов.

Сводка некоторых данных по сопоставлению характеристик случайной составляющей погрешностей непосредственно измеряемых площадей пиков хлорбензола и их отношений к площадям пиков дополнительного внутреннего стандарта (1,2,4-триметилбензол) в условиях низкой воспроизводимости дозирования проб приведена в табл. 4. Ее последняя строка иллюстрирует уменьшение неопределенности результатов измерений абсолютных и относительных площадей пиков, выражаемое отношениями их коэффициентов вариации, которое в рассматриваемом примере (при больших неконтролируемых потерях проб в ходе дозирования) достигает 35-38. При «нормальной» воспроизводимости дозирования такой выигрыш несколько меньше, но, тем не менее, достигает 5-10 [12-15].

Таблица 4. Сравнение погрешностей непосредственно измеряемых площадей пиков хлорбензола и их отношений к площадям пиков дополнительного стандарта $(1,2,4-$ триметилбензол) в условиях низкой воспроизводимости дозирования проб

\begin{tabular}{|c|c|c|}
\hline Характеристика массива данных & $\begin{array}{c}\text { Раствор с условно не- } \\
\text { известной концентра- } \\
\text { цией аналита }\end{array}$ & Раствор внешнего стандарта \\
\hline$S_{\mathrm{x}} \pm s\left(S_{\mathrm{x}}\right)$ & $19670 \pm 10351$ & $63274 \pm 20185$ \\
\hline$S_{\text {доп.ст }} \pm s\left(S_{\text {доп.ст }}\right)$ & $22107 \pm 11690$ & $34817 \pm 10900$ \\
\hline$\left(S_{\mathrm{x}} / S_{\text {доп.ст }} \pm s\left(S_{\mathrm{x}} / S_{\text {доп.ст }}\right)\right.$ & $0.89 \pm 0.01$ & $1.81 \pm 0.02$ \\
\hline$\delta_{1}=\delta\left(S_{\mathrm{x}}\right), \%$ & 53 & 32 \\
\hline$\delta\left(S_{\text {доп.ст }}\right) \%$ & 53 & 31 \\
\hline$\delta_{2}=\delta\left(S_{\mathrm{x}} / S_{\text {доп.ст }}\right) \%$ & 1.4 & 32 \\
\hline $\begin{array}{c}\text { Мера уменьшения степени неопре- } \\
\text { деленности результатов, }\left(\delta_{1} / \delta_{2}\right)\end{array}$ & 38 & \\
\hline
\end{tabular}

Аналогична по структуре табл. 5 характеризует уменьшение степени неопределенности результатов (последняя строка таблицы) в результате использования в качестве дополнительного стандарта растворителя (2-пропанол), которое также достаточно велико (10-20).

Использование дополнительных стандартов закономерно влечет за собой трансформацию расчетных соотношений. Так, основное расчетное уравнение способа внешнего стандарта (1) приобретает следующий вид:

$$
C_{x}=C_{c m}\left[\frac{S_{x}}{S_{\text {доn.cm }(x)}}\right] \div\left[\frac{S_{c m}}{S_{\text {доn.cm }(\mathrm{cm})}}\right] \text { при } M_{\text {доп.ст(х) }}=M_{\text {доп.ст(ст) }}
$$

где $C_{\mathrm{x}}, C_{\text {ст }}, S_{\mathrm{x}}$ и $S_{\text {ст }}-$ те же, что и в формуле $(1) ; S_{\text {доп.ст(х) }}$ и $S_{\text {доп.ст(ст) }}-$ площади пиков дополнительного стандарта в анализируемом образце $(x)$ и внешнем стандарте (ст).

Выполнение условия $M_{\text {доп.ст(х) }}=M_{\text {доп.ст(ст) }}$ должно быть обеспечено экспериментально, иначе это может привести к увеличению систематических погрешностей определений. При всей простоте соотношения (4) нельзя не прокомментировать его главную особенность. Если объемы образцов равны, то условие $M_{\text {доп.ст(х) }}=M_{\text {доп.ст(ст) }}$ эквивалентно условию $C_{\text {доп.ст(х) }}=C_{\text {доп.ст(ст) }}$, но тогда $S_{\text {доп.ст(х) }} \approx S_{\text {доп.ст(ст) }}$ и, формально, равные значения площадей пиков можно сократить. Это должно было бы превратить соотношение (4) в известную формулу для обычного метода внешнего стандарта (2), 
однако именно эта математическая операция недопустима, так как не отражает последовательность экспериментальных действий. Дело в том, что при использовании формулы (4) сначала должны быть вычислены и усреднены значения отношений

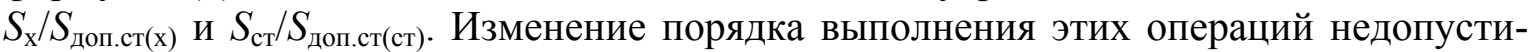
мо. По аналогии с формулой (3) соотношение (4) можно переписать следующим образом:

$$
C_{x}=C_{c m}\left\langle\frac{S_{x}}{S_{\text {доn.cm }(x)}}\right\rangle \div\left\langle\frac{S_{c m}}{S_{\text {доn.cm }(c m)}}\right\rangle \text { при } M_{\text {доп.ст(х) }}=M_{\text {доп.ст(ст) }}
$$

Таблица 5. Сравнение погрешностей непосредственно измеряемых площадей пиков хлорбензола в модельных образцах и их отношений к площадям пиков растворителя (2-пропанол) в условиях низкой воспроизводимости дозирования проб

\begin{tabular}{|c|c|c|}
\hline Характеристика массива данных & $\begin{array}{c}\text { Раствор с условно } \\
\text { неизвестной кон- } \\
\text { центрацией } \\
\text { аналита }\end{array}$ & $\begin{array}{c}\text { Раствор внешнего } \\
\text { стандарта }\end{array}$ \\
\hline$S_{\text {раств }} \pm s\left(S_{\text {раств }}\right) \times 10^{-5}$ & $3.4 \pm 1.7$ & $5.3 \pm 1.6$ \\
\hline$\delta_{1}=\delta\left(S_{\mathrm{x}}\right) \%$ & 53 & 32 \\
\hline$\delta\left(S_{\text {раств }) \%}\right) \%$ & 50 & 1.6 \\
\hline$\delta_{2}=\delta\left(S_{\mathrm{x}} / S_{\text {раств }}\right) \%$ & 5.4 & $8.4 \pm 0.1$ \\
\hline$\left(S_{\mathrm{x}} / S_{\text {раств }}\right) \pm s\left(S_{\mathrm{x}} / S_{\text {раств }}\right)$ & $17.5 \pm 0.9$ & 20 \\
\hline $\begin{array}{c}\text { Мера уменьшения степени неопределенности } \\
\text { результатов, }\left(\delta_{1} / \delta_{2}\right)\end{array}$ & 9.8 & \\
\hline
\end{tabular}

Применение дополнительных стандартов изменяет также соотношение для оценки относительных погрешностей результатов определений методом внешнего стандарта; в нем фигурируют коэффициенты вариации не абсолютных площадей пиков, а их отношений:

$$
\delta C_{x} \approx\left[\delta\left(\frac{S_{x}}{S_{\text {доn.cm }(x)}}\right)^{2}+\delta\left(\frac{S_{c m}}{S_{\text {доn.cm }(\mathrm{cm})}}\right)^{2}\right]^{1 / 2}
$$

Способ стандартной добавки. Основное уравнение этого способа, записанное с использованием символов усреднения, имеет вид:

$$
M_{x}=M_{\text {доб }} \frac{\left\langle S_{x}\right\rangle}{\left\langle S_{x+д о б}\right\rangle-\left\langle S_{x}\right\rangle}=\frac{M_{\text {доб }}}{\left(\left\langle S_{x+д о б}\right\rangle /\left\langle S_{x}\right\rangle-1\right)}
$$

где $M_{\mathrm{x}}$ и $M_{\text {доб }}$ - содержание определяемого компонента в образце и масса стандартной добавки, $\left\langle S_{\mathrm{x}}>\right.$ и $\left\langle S_{\mathrm{x}+д о б}>-\right.$ средние значения площадей пиков компонента до и после добавки.

В этом способе анализируют один и тот же образец до и после добавки определяемого компонента. В модифицированном варианте стандартной добавки дополнительный стандарт должен быть введен в образец предварительно с целью замены абсолютных величин $\left(S_{\mathrm{i}}\right)$ отношениями $\left(S_{\mathrm{i}} / S_{\text {доп.станд }}\right)$. Основное расчетное соотношение при этом приобретает следующий вид:

$$
M_{x}=M_{\text {доб }} \frac{\left\langle S_{x} / S_{\text {доn.cm }}\right\rangle}{\left\langle S_{x+\partial о \sigma} / S_{\text {доg.cm }}\right\rangle-\left\langle S_{x} / S_{\text {доn.cm }}\right\rangle}
$$

Как и в соотношении (5), здесь сначала вычисляют отношения площадей, которые до подстановки в формулу (8) усредняют. Несколько усложняется соотношение для оценки относительных погрешностей результатов определений, хотя изменения касаются лишь замены абсолютных площадей пиков соответствующими относительными величинами: 


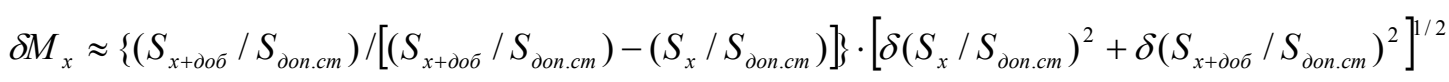

В табл. 6 сопоставлены результаты анализа модельных образцов с концентра-

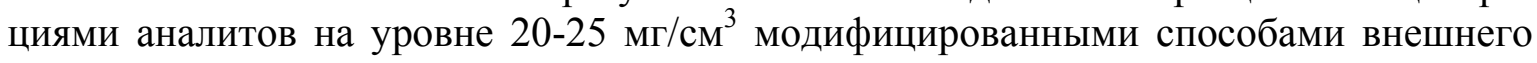
стандарта и стандартной добавки.

Таблица 6. Сравнение результатов количественного определения хлорбензола (I) и изопентанола (II) «стандартными» и модифицированными способами внешнего стандарта и стандартной добавки при различной воспроизводимости дозирования проб.

\begin{tabular}{|c|c|c|c|c|c|}
\hline \multirow{2}{*}{$\begin{array}{c}\text { Вариант } \\
\text { определений }\end{array}$} & \multirow{2}{*}{ 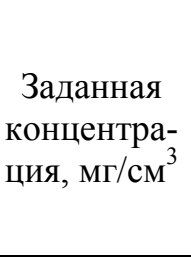 } & \multicolumn{2}{|c|}{$\begin{array}{c}\text { Результат определений мето- } \\
\text { дом внешнего стандарта, } C \pm \\
s(C), \text { мг/см}{ }^{3}\end{array}$} & \multicolumn{2}{|c|}{$\begin{array}{c}\text { Результат определений ме- } \\
\text { тодом стандартной добавки, } \\
C \pm s(C), \text { мг/см }{ }^{3}\end{array}$} \\
\hline & & $\begin{array}{c}\text { Без допол- } \\
\text { нительного } \\
\text { стандарта }\end{array}$ & $\begin{array}{l}\text { С дополни- } \\
\text { тельным } \\
\text { стандартом }\end{array}$ & $\begin{array}{c}\text { Без допол- } \\
\text { нительного } \\
\text { стандарта }\end{array}$ & $\begin{array}{l}\text { С дополни- } \\
\text { тельным } \\
\text { стандартом }\end{array}$ \\
\hline \multirow{2}{*}{$\begin{array}{c}\text { Низкая } \\
\text { воспроизводимость } \\
\text { дозирования } \\
\end{array}$} & (I) 26.4 & $16.0 \pm 9.9$ & $25.3 \pm 0.4$ & $11 \pm 10$ & $24.2 \pm 0.8$ \\
\hline & (II) 19.3 & $28 \pm 12$ & $17.7 \pm 0.9$ & $56 \pm 94$ & $16.2 \pm 1.6$ \\
\hline $\begin{array}{c}\text { «Нормальная» вос- } \\
\text { производимость } \\
\text { дозирования }\end{array}$ & (II) 19.3 & $19.8 \pm 1.1$ & $18.3 \pm 0.1$ & $20.4 \pm 2.4$ & $17.3 \pm 0.2$ \\
\hline
\end{tabular}

*Курсивом набраны корректные результаты определений.

Таблица 7. Сравнение результатов количественного определения хлорбензола (I) и изопентилового спирта (II) в модельных образцах способами внешнего стандарта и стандартной добавки «традиционным» способом и при использовании 2-пропанола (растворитель) в качестве дополнительного стандарта в условиях различной воспроизводимости дозирования.

\begin{tabular}{|c|c|c|c|c|c|}
\hline & \multirow{2}{*}{ 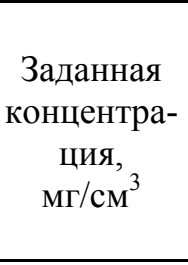 } & \multicolumn{2}{|c|}{$\begin{array}{c}\text { Метод внешнего стандарта, } \\
C \pm s(C), \mathrm{Mr}^{3} \mathrm{~cm}^{3}\end{array}$} & \multicolumn{2}{|c|}{$\begin{array}{c}\text { Метод стандартной добавки, } \\
C \pm s(C), \mathrm{Mr} / \mathrm{cm}^{3}\end{array}$} \\
\hline & & $\begin{array}{c}\text { Без допол- } \\
\text { нительного } \\
\text { стандарта }\end{array}$ & $\begin{array}{c}\text { Растворитель в } \\
\text { качестве } \\
\text { дополнительного } \\
\text { стандарта }\end{array}$ & $\begin{array}{c}\text { Без допол- } \\
\text { нительного } \\
\text { стандарта }\end{array}$ & $\begin{array}{c}\text { Растворитель } \\
\text { в качестве до- } \\
\text { полнитель- } \\
\text { ного стандарта }\end{array}$ \\
\hline \multirow{2}{*}{$\begin{array}{c}\text { Низкая } \\
\text { воспроизводимость } \\
\text { дозирования }\end{array}$} & 26.4 (I) & $16.0 \pm 9.9$ & $25.4 \pm 1.4$ & $11 \pm 10$ & $24.4 \pm 2.5$ \\
\hline & 19.3 (II) & $28 \pm 12$ & $18.9 \pm 0.9$ & $56 \pm 94$ & $18.5 \pm 1.7$ \\
\hline $\begin{array}{c}\text { «Нормальная» } \\
\text { воспроизводимость } \\
\text { дозирования }\end{array}$ & 19.3 (II) & $19.8 \pm 1.1$ & $18.9 \pm 0.3$ & $20.4 \pm 2.4$ & $18.6 \pm 0.6$ \\
\hline
\end{tabular}

*Курсивом набраны корректные результаты определений. **Количество примеров при необходимости может быть увеличено.

При низкой воспроизводимости дозирования проб относительные стандартные отклонения результатов закономерно неприемлемы и достигают 40-170\% при систематических погрешностях (отклонения «введено-найдено») от -60 до $+190 \%$ отн. Использование дополнительного стандарта в методе внешнего стандарта обеспечивает уменьшение коэффициентов вариации результатов до 0.1-0.9 \% при различиях «введено-найдено» 1.1-1.6 \%. Для метода стандартной добавки аналогичные характеристики закономерно несколько хуже: коэффициенты вариации 0.2-1.6 \% при отклонениях «введено-найдено» 2.2-3.1 \%. В табл. 7 приведены аналогичные харак- 
теристики этих же способов при использовании в качестве внутреннего стандарта растворителя (2-пропанол).

Особенности способа абсолютной градуировки. Один из важнейших способов количественного хроматографического анализа - абсолютной градуировки - также основан на измерении абсолютных значений площадей пиков $\left(S_{\mathrm{i}}\right)$ и, следовательно, может быть модифицирован за счет их замены относительными величинами $\left(S_{\mathrm{i}} /\right.$

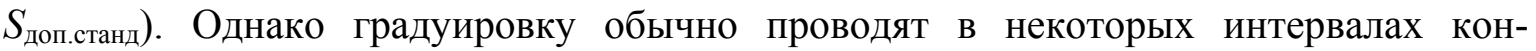
центраций аналитов. Это может привести к использованию нескольких различных по величине добавок дополнительных стандартов, что ведет к некоторому усложнению как экспериментальных операций, так и обработки данных [14]. Относительные площади пиков, соответствующие каждому значению массы аналита, должны быть сопоставлены с одинаковыми или разными количествами дополнительных стандартов, рассматриваемыми, например, в виде отношений их концентраций к минимальной величине $C_{\text {доп.ст }}(1)$, т.е. $C_{\text {доп.ст }}(\mathrm{i}) / C_{\text {доп.ст }}(1)$. Для задания неодинаковых количеств дополнительных стандартов удобнее всего использовать различные объемы одного и того же раствора, поскольку $C_{\text {доп.ст }}(\mathrm{i}) / C_{\text {доп.ст }}(1)=k\left[V_{\text {доп.ст }}(\mathrm{i}) / V_{\text {доп.ст }}(1)\right], \quad$ где $k(v)=\left(v_{\text {раств }}+v_{\text {доп.ст }}\right) /\left(v_{\text {раств }}+v_{\mathrm{x}}+v_{\text {доп.ст }}\right)-$ коэффициент, учитывающий изменение объемов растворов при их смешении.

$$
C_{x}=k(v) \cdot a \cdot\left[\left\langle S_{x}(i) / S_{\text {доn.cm }}(i)\right\rangle\right] \cdot\left[C_{\text {доn.cm }}(i) / C_{\text {доп.cm }}(1)\right]+b,
$$

где $<S_{\mathrm{x}}(\mathrm{i}) / S_{\text {доп.ст }}(\mathrm{i})>$ - средние значения отношений площадей пиков, соответствующие отношению $C_{\text {доп.ст }}(\mathrm{i}) / C_{\text {доп.ст }}(1)$, коэффициенты уравнения линейной регрессии $a$ и $b$ вычисляют методом наименьших квадратов.

Более детально повышение точности результатов при использовании модифицированного способа абсолютной градуировки рассмотрено в работе [14].

Комментарии к способу внутреннего стандарта. Количественный анализ способом внутреннего стандарта основан на введении в анализируемые образцы (обычно растворы) известных количеств дополнительного компонента - стандарта. Этим методом можно определять как концентрации целевых компонентов в образцах $\left(C_{\mathrm{x}}\right)$, так и их суммарные количества $\left(M_{\mathrm{x}}\right)$. Однако небезынтересно заметить, что в современной литературе (см., например $[1-4,20])$ при представлении расчетных формул чаще всего не указывают последовательности усреднения входящих в них величин. В корректной же записи должно быть отмечено, что сначала (для каждой хроматограммы) вычисляют отношения $\left(S_{\mathrm{x}} / S_{\text {станд }}\right)$, после чего их усредняют [с оценкой стандартных отклонений не абсолютных, а относительных площадей пиков, $\left.s\left(S_{\mathrm{x}} / S_{\text {станд }}\right)\right]$, и только после этого средние значения подставляют в формулу (11):

$$
C_{x} \cdot\left(M_{x}\right)=f_{x / \text { станд }} \cdot C_{\text {станд }} \cdot\left(M_{\text {станл }}\right) \cdot\left\langle S_{x} / S_{\text {станд }}\right\rangle,
$$

где $S_{\mathrm{x}}$ и $S_{\text {станд }}$ - площади пиков определяемого компонента и внутреннего стандарта, $C_{\text {станд }}\left(M_{\text {станд }}\right)$ - заданные концентрация или масса внутреннего стандарта, $f_{\mathrm{x} / \text { станд }}-$ градуировочный коэффициент аналита относительно выбранного стандарта (для компенсации различий чувствительности используемых детекторов к разным соединениям).

Таким образом, процедура обработки экспериментальных данных в способе внутреннего стандарта идентична вычислениям при использовании дополнительных стандартов, что делает их применение в этом способе нецелесообразным. Однако уточнение последовательности обработки данных позволяет сформулировать важные выводы относительно соотношений для оценки случайной составляющей погрешности этого варианта количественного хроматографического анализа.

Если проигнорировать первичное усреднение отношений площадей пиков $<S_{\mathrm{x}} / S_{\text {станд }}>$ в соотношении (11), то для оценки случайной составляющей формальным 
дифференцированием уравнения (11) получаем соотношение (11), упомянутое, в частности, в справочнике [22]:

$$
\delta\left[C_{x}\left(M_{x}\right)\right] \approx\left[\delta\left(S_{x}\right)^{2}+\delta\left(S_{\text {станд }}\right)^{2}+\delta\left(f_{x / \text { станд }}\right)^{2}\right]^{1 / 2}
$$

Однако оно принципиально некорректно, поскольку по сравнению с формулой оценки случайных погрешностей способа внешнего стандарта (3) содержит дополнительное слагаемое $\delta\left(f_{\mathrm{x} / \text { станд }}\right)$. На самом же деле случайная составляющая погрешностей способа внутреннего стандарта не может превышать эту характеристику способа внешнего стандарта, так что формула (11) должна быть пересмотрена с учетом вычисления отношений $\left(S_{\mathrm{x}} / S_{\text {станд }}\right)$ :

$$
\delta\left[C_{x}\left(M_{x}\right)\right] \approx\left[\delta\left(S_{x} / S_{\text {станд }}\right)^{2}+\delta\left(f_{\text {x/станд }}\right)^{2}\right]^{1 / 2}
$$

В формуле (13) присутствует еще один сомножитель, а именно относительное стандартное отклонение градуировочного коэффициента аналита относительно внутреннего стандарта $\left(f_{\text {хсстанд }}\right)$. Экспериментальное определение $f_{\mathrm{x} / \text { станд }}$ основано на анализе специально приготовленных образцов с известными содержаниями аналита и стандарта:

$$
f_{\text {x/станд }}=\left[C_{x}^{\prime} \cdot\left(M_{x}^{\prime}\right) / C_{\text {станд }}^{\prime} \cdot\left(M_{\text {станд }}^{\prime}\right) k S_{\text {станд }}^{\prime} / S_{x}^{\prime}\right\rangle
$$

Штрихи в обозначениях переменных в формуле (14) соответствуют их определению в градуировочных растворах. Как и в формуле (11) вместо концентраций $C_{\mathrm{x}}{ }^{\prime}$ и $C_{\text {станд }}$ 'здесь могут быть использованы суммарные количества компонентов, $M_{\mathrm{x}}{ }^{\prime}$

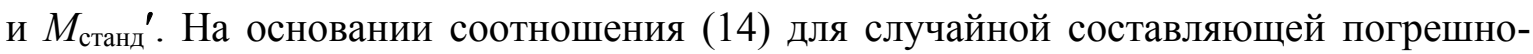
стей градуировочных коэффициентов можно записать:

$$
\delta f_{x / \text { станд }}=\delta\left(S_{\text {станд }}^{\prime} / S_{x}^{\prime}\right)
$$

Подстановка (15) в (13) дает соотношение для случайной составляющей погрешностей способа внутреннего стандарта, существенно отличающееся от формулы (12):

$$
\delta\left[C_{x}^{\prime} \cdot\left(M_{x}^{\prime}\right)\right] \approx\left[\delta\left(S_{x} / S_{\text {станд }}\right)^{2}+\delta\left(S_{\text {станд }}^{\prime} / S_{x}^{\prime}\right)^{2}\right]^{1 / 2}
$$

Практически все соотношения для модифицированных способов количественного анализа могут быть использованы не с площадями, а с высотами хроматографических пиков. Проверка этого варианта показала, что точность определений в этом случае несколько хуже, что связано с дополнительными факторами, влияющими на случайный разброс высот пиков.

\section{Эксперимент}

Приготовление модельных образцов. Использовали двухкомпонентные растворы, один из компонентов которых условно считали определяемым, а второй рассматривали как дополнительный стандарт. Растворы смесей хлорбензола (ч, для хроматографии, условно определяемый компонент, 50 и 100 мкл) и 1,2,4-триметилбензола (ч, для хроматографии, дополнительный стандарт, 50 мкл) в 2 см $^{3} 2$-пропанола (Вектон, Санкт-Петербург) готовили по объему (образец № 1). Образец № 2 состоял из изопентанола (условно определяемый компонент, 50 и 100 мкл) и 1-гексанола (ч, дополнительный стандарт, 50 мкл) в таком же объеме 2-пропанола. Определяемые компоненты и стандарты дозировали шприцем МШ-50, растворитель - медицинским шприцем объемом $2 \mathrm{~cm}^{3}$. Концентрации аналитов и стандартов пересчитывали в массо-объемные единицы (мг/ $\left.\mathrm{cm}^{3}\right)$ с учетом их плотностей при комнатной температуре. 
Растворы различных соединений (анизол, 1-гептанол, бутилацетат, изопропилбутаноат, бутилбутират, ацетофенон, и другие) для характеристики модифицированного способа абсолютной градуировки готовили, добавляя 10, 25, 50, 100, 200 и 400 мкл компонента $\left(v_{x}\right)$ и 50 или 250 мкл стандарта $\left(v_{\text {ст }}\right)$ к $2 \mathrm{~cm}^{3}$ изопропанола $\left(v_{\text {раств}}\right)$ [14]. Для каждого из образцов вычисляли значение поправочного коэффициента, учитывающего изменение их объема при смешении компонентов

$$
k(v)=\left(v_{\text {pacms }}+v_{\text {доn.cm }}\right) /\left(v_{\text {pacms }}+v_{x}+v_{\text {доn.cm }}\right)
$$

Газохроматографический анализ образцов проводили на хроматографе Кристалл 5000.2 с пламенно-ионизационным детектором (ПИД) с использованием двух WCOT капиллярных колонок: а) длиной 10 м и внутренним диаметром 0.53 мм со стандартной неполярной полидиметилсилоксановой неподвижной фазой BPX-1 (SGE Analytical Science, Австралия), толщина пленки фазы 2.65 мкм и б) Optima-1 (полидиметилсилоксан, Macherey Nagel, Германия) длиной 25 м и внутренним диаметром 0.32 мм, толщина пленки фазы 0.35 мкм. Режимы анализа: изотермический, $120-130^{\circ} \mathrm{C}$ (a), программирование температуры от 90 до $146^{\circ} \mathrm{C}$ со скоростью 8 град/мин и далее 54 град/мин до $200^{\circ} \mathrm{C}$ (прогрев колонки для удаления возможных примесей) (б); газ-носитель азот, расход 4.0 (а) и $1.0 \mathrm{~cm}^{3} /$ мин (б); деление потока при дозировании проб $5: 1$ (а) и $10: 1$ (б); температура испарителя 150 (а) и $200^{\circ} \mathrm{C}$ (б); температура детектора $200^{\circ} \mathrm{C}$. Объем дозируемых проб во всех случаях 0.5 мкл. При моделировании низкой воспроизводимости дозирования использовали микрошприц МШ-1 (АО «Цвет», Дзержинск), активно эксплуатировавшийся в течение приблизительно года в Ресурсном образовательном центре по направлению «Химия» при Институте химии СПбГУ. Моделирование «нормальной» воспроизводимости дозирования проводили с использованием не использовавшегося ранее микрошприца Газохром-101, Статистическую обработку данных и построение гистограмм проводили с использованием программного обеспечения Excel (Microsoft Office 2010) и Origin (версии 4.1 и 8.1). Число параллельных определений в случае низкой воспроизводимости составляло не менее 20, при «нормальной» - не менее пяти.

Особенности применения модифицированных способов количественного анализа проверены при выполнении практических работ по курсу «Газовая хроматография» студентами Института химии СПбГУ. Немаловажно отметить такое их преимущество как существенная экономия времени за счет исключения необходимости достижения приемлемой воспроизводимости результатов, поскольку практически все пробы соответствуют этому критерию.

\section{Заключение}

Одним из основных источников погрешностей количественного газохроматографического анализа являются трудно выявляемые частичные потери проб при их дозировании в хроматографические колонки. Если этот источник по тем или иным причинам не удается устранить, он может быть эффективно скомпенсирован за счет добавки к анализируемым образцам дополнительных стандартов с целью замены абсолютных площадей пиков $\left(S_{\mathrm{i}}\right)$ более воспроизводимыми относительными величинами $\left(S_{\mathrm{i}} / S_{\text {доп.станд }}\right)$.

Требования к дополнительным стандартам лишь частично совпадают с требованиями к внутренним стандартам. Основными отличиями являются отсутствие необходимости задания их количеств (концентраций) в образцах и исключение предварительного определения градуировочных коэффициентов. 
Максимальной простотой и наибольшей эффективностью (степень уменьшения погрешностей результатов определений) применение дополнительных стандартов характеризуется в способах внешнего стандарта и стандартной добавки. В способе абсолютной градуировки оно несколько более трудоемко, а в способах внутреннего стандарта и внутренней нормализации не дает никаких преимуществ.

Работа выполнена с использованием оборудования ресурсного ичентра по направлению «Химия» Института Химии Санкт-Петербургского государственного университета. Авторы выражают благодарность сотрудникам иентра за содействие.

\section{Список литературы}

1. Зенкевич И.Г., Прокофьев Д.В. // Тез. докл. V Всерос. Симп. «Кинетика и динамика обменных процессов». Сочи. С. 74-76.

2. Новак Й. Количественный анализ методом газовой хроматографии. Пер. с англ. М: Мир. 1978. 180 с.

3. Руководство по газовой хроматографии. Под ред. Э. Лейбница и Х.Г. Штруппе. Пер. с нем. М. Мир. 1988. Т. 2. 510 с.

4. Столяров Б.В., Савинов И.М., Витенберг А.Г., Карцова Л.А. и др. Практическая газовая и жидкостная хроматография. СПб: Изд. СПб ун-та. 2002. 616 с.

5. Practical Gas Chromatography. A Comprehensive Reference. Ed. K. Dettmer-Wilde, W. Engewald. Heidelberg: Springer, 2014. 902 p.

6. Sparkman O.D., Penton Z., Kitson F. Gas Chromatography and Mass Spectrometry. New York: Academic Press. 2011. 632 p.

7. Апраксин В.Ф. Количественный газохроматографический анализ. Методические указания. СПб. Изд. СПХФА. 1999. 25 с.

8. Cuadros-Rodrigues J., Bagur-Gonsalez M.G., Sanchez-Vinas M., Gonsalez-Casado A. et al. // J. Chromatogr. A. 2007. Vol. 1158. pp. $33-46$.

9. Usher K.M., Hansen S.W., Amoo J.S., Bernstein A.P. et al., $L C$-GC. 2015. No 6. pp. 113.

10. Raposo F. // Trends Anal. Chem. 2016. Vol. 72. pp. 167-185.

11. Asnin L.D. // Trends Anal. Chem. 2016. No 1. pp. 51-62.

12. Зенкевич И.Г., Прокофьев Д.В. // Аналитика и контроль. 2015. Т. 19. № 4. С. 302309.
13. Зенкевич И.Г., Прокофьев Д.В. // Аналитика и контроль. 2016. Т. 20. № 2. С. 147153.

14. Прокофьев Д.В., Зенкевич И.Г. // Вестн. СПбГУ. Сер. физ.-хим. 2016. Т. 3(61). Вып. 3. С. 337-344.

15. Зенкевич И.Г., Прокофьев Д.В. // Заводская лаборатория. Диагностика материалов. 2016. Т. 82. № 11. С. 5-11.

16. Черепица С.В., Бычков С.М., Коваленко А.Н., Мазаник А.Л. и др. // Журн. аналит. химии. 2003. Т. 58. № 4. С. 416-420.

17. Черепица С.В., Бычков С.М., Коваленко А.Н., Мазанник А.Л. и др. // Зав. лаборатория. Диагностика материалов. 2003. Т. 69. № 2. C. 22-25.

18. Черепица С.В., Бычков С.М., Гациха С.В., Коваленко А.Н. и др. // Партнеры и конкуренты. Лабораториум. 2004. № 8. С. 35-39.

19. Charapitsa S.V., Kavalenka A.N., Kulevich N.V., Makoed N.M. et al. // J. Agric. Food Chem. 2013. Vol. 61. pp. 2950-2956.

20. Царев Н.И., Царев В.И., Катраков И.Б. Практическая газовая хроматография. Барнаул: Изд. Алтайск. ГУ. 2000. 156 с.

21. The NIST 14 Mass Spectral Library (NIST14/2014/EPA/NIH). Software/Data Version (NIST14); NIST Standard Reference Database, Number 69, 2014. National Institute of Standards and Technology, Gaithersburg, MD 20899; http://webbook.nist.gov (дата обращения: январь 2017 г.).

22. Мариничев А.Н., Турбович М.Л., Зенкевич И.Г. Физико-химические расчеты на микро-ЭВМ. Справочник. Л: Химия, 1990. $254 \mathrm{c}$. 


\section{References}

1. Zenkevich I.G., Prokof'ev D.V., Abstr. V all-Russ. Symp. "Kinetika I dinamika obmennykh processov", Sochi, 2016. No 22. pp. 7476. [in Russian].

2. Novak J. Quantitative Analysis by Gas Chromatography. New York, Marcel Dekker Inc., 1975, $180 \mathrm{p}$.

3. Handbuch der Gaschromatographie. Ed. E.Leibnitz, H.G. Struppe. Leipzig: Akademische Verlag, 1984, $510 \mathrm{p}$.

4. Stolarov B.V., Savinov I.M., Vitenberg A.G., Kartsova L.A. et al., Prakticheskaya gazovaya i zhidkostnaya khromatografia Practical gas and liquid chromatography . St. Petersburg. University Press, 2002. 610 p. [in Russian]

5. Practical Gas Chromatography. A Comprehensive Reference. Ed. K. Dettmer-Wilde, W. Engewald. Heidelberg: Springer, 2014, 902 p.

6. Sparkman O.D., Penton Z., Kitson F. Gas Chromatography and Mass Spectrometry. New York: Academic Press, 2011, 632 p.

7. Apraksin V.F. Kolichestvennyi gazokhromatograficheskii analiz. Metodicheskie ukazaniya. St. Petersburg: St. Petersburg ChemicalPharmaceutical Academy Press, 1999, 25 p. [in Russian]

8. Cuadros-Rodrigues J., Bagur-Gonsalez M.G., Sanchez-Vinas M., Gonsalez-Casado A. et al., J. Chromatogr. A, 2007, Vol. 1158, pp. 33-46. DOI: 10.1016/j.chroma.2007.03.030.

9. Usher K.M., Hansen S.W., Amoo J.S., Bernstein A.P., et al., $L C-G C, 2015$, No 6, pp. 1-13.

10. Raposo F., Trends Anal. Chem., 2016, Vol. 72, pp. 167-185. DOI: 10.1016/j.trac.2015.12.006.

11. Asnin L.D., Trends Anal. Chem., 2016, No. 1, pp. 51-62. DOI: 10.1016/j.trac.2015.01.006.

12. Zenkevich I.G., Prokof'ev D.V., Analitika $i$ kontrol'. 2015, Vol. 19, No 4, pp. 302-309. [in

Зенкевич Игорь Георгиевич - д.х.н., профессор кафедры органической химии Института химии СанктПетербургского государственного университета, Санкт-Петербург, тел. (812) 428-4045

Прокофьев Денис Витальевич - студент 2-го курса магистратуры Института химии Санкт-Петербургского государственного университета, Санкт-Петербург, тел. (812) 428-4045
Russian]. DOI

$10.15826 /$

analitika.2015.19.4.007.

13. Zenkevich I.G., Prokof'ev D.V., Analitika i kontrol', 2016, Vol. 20, No 2, pp. 147-153. [in Russian].

DOI: 10.15826/analitika.2016.20.2.005.

14. Prokof'ev D.V., Zenkevich I.G., Vestnik SPbGU. Ser. phis-khim., 2016, Vol. 3(61), No. 3, pp. 337-344. [in Russian]

15. Zenkevich I.G., Prokof'ev D.V., Zavodskaia Laboratoria. Diagnostika materialov. 2016, Vol. 82, No 11, pp. 5-11. [in Russian]

16. Cherepitsa S.V., Bychkov S.M., Kovalenko A.N., Mazanik A.L. et al., Zhurn. Analit. Khimii. Vol. 58, No 4, pp. 416-420. [in Russian]

17. Cherepitsa S.V., Bychkov S.M., Kovalenko A.N., Mazannik A.L. et al., Zavodskaya laboratoria. Diagnostika materialov. 2003, Vol. 69, No 2, pp. 22-25. [in Russian]

18. Cherepitsa S.V., Bychkov S.M., Gatsikha S.V., Kovalenko A.N. et al., Partnery i konkurenty. Laboratorium, 2004, No 8, pp. 35-39. [in Russian]

19. Charapitsa S.V., Kavalenka A.N., Kulevich N.V., Makoed N.M. et al., J. Agric. Food Chem., 2013, Vol. 61, pp. 2950-2956. DOI: 10.1021/jf3044956.

20. Tsarev N.I., Tsarev V.I., Katrakov I.B. Prakticheskaia gazovaia khromatografia. Barnaul: Altai State Univ., Press, 2000, 156 p. [in Russian]

21. The NIST 14 Mass Spectral Library (NIST14/2014/EPA/NIH). Software/Data Version (NIST14); NIST Standard Reference Database, Number 69, 2014. National Institute of Standards and Technology, Gaithersburg, MD 20899; http://webbook.nist.gov (accessed: January 2017).

22. Marinichev A.N., Turbovich M.L., Zenkevich I.G. Fisiko-khimicheskie raschety na mikro-EVM. Spravochnik. Leningrad: Khimia Publ., 1990, 254 p. [in Russian]

Zenkevich Igor G. - Dr. Sci., Professor, Chair of Organic Chemistry, Institute for Chemistry, St. Petersburg State University, St. Petersburg, E-mail: izenkevich@yandex.ru

Prokofiev Denis V. - Magister, Institute for Chemistry, St. Petersburg State University, St. Petersburg, E-mail: deniss-prokofev@yandex.ru 